\title{
Assessing the risk of default propagation in interconnected sectoral financial networks
}

\author{
Adrià Barja' , Alejandro Martínez ${ }^{1}$, Alex Arenas², Pablo Fleurquin' ${ }^{1}$, Jordi Nin³ ${ }^{3}$, José J. Ramasco ${ }^{4 *}$ (D) and \\ Elena Tomás ${ }^{1}$
}

\section{"Correspondence:}

jramasco@ifisc.uib-csic.es

${ }^{4}$ Instituto de Física Interdisciplinar y Sistemas Complejos IFISC

(CSIC-UIB), Palma de Mallorca, Spain

Full list of author information is

available at the end of the article

\section{Springer}

\begin{abstract}
Systemic risk of financial institutions and sectoral companies relies on their inter-dependencies. The inter-connectivity of the financial networks has proven to be crucial to understand the propagation of default, as it plays a central role to assess the impact of single default events in the full system. Here, we take advantage of complex network theory to shed light on the mechanisms behind default propagation. Using real data from the BBVA, the second largest bank in Spain, we extract a financial network from customer-supplier transactions among more than 140,000 companies, and their economic flows. Then, we introduce a computational model, inspired by the probabilities of default contagion, that allow us to obtain the main statistics of default diffusion given the network structure at individual and system levels. Our results show the exposure of different sectors to default cascades, therefore allowing for a quantification and ranking of sectors accordingly. This information is relevant to propose countermeasures to default propagation in specific scenarios.
\end{abstract}

Keywords: Financial networks; Default analysis; Financial sector analytics; SIS propagation models; Complex systems

\section{Introduction}

Interconnected financial networks are the fabric where economic agents from different sectors operate. One of the main challenges we face nowadays on financial networks is assessing systemic risk [1-3]. In the literature, systemic risk is defined as the probability of having large cascades of entangled economic events. Such cascades are triggered by causes that range from exogenous shocks to endogenous defaults. Besides, the succession of several defaults can jeopardize the full system because network financial inter-dependencies act as an economic sounding board. The interplay between the topology of the underlying interaction network and the easiness with which events propagate have proven to be essential to understand the proportion of the financial system affected by default avalanches and to assess the systemic risk [4].

Avalanches in financial systems are understood as dynamical processes that correlate individual economic states of the agents when a stress event materializes. This process resembles epidemic spreading in networks [5]. Under a simplifying assumption and to better explore the network potential for risk transmission, we model them in a similar way as epidemic spreading as was recently done in [6-8]. This is an oversimplification, the basic

(c) The Author(s) 2019. This article is distributed under the terms of the Creative Commons Attribution 4.0 International License (http://creativecommons.org/licenses/by/4.0/), which permits unrestricted use, distribution, and reproduction in any medium, provided you give appropriate credit to the original author(s) and the source, provide a link to the Creative Commons license, and indicate if changes were made. 
mechanisms of default propagation and epidemic spreading have similarities as branching process and chain reactions. Still in economic systems, especially those involving large companies such as banks, there are other mechanisms that may delay or even prevent the final default. Given the simplicity of the epidemic models and the fact that our networks are mostly formed by small and medium enterprises, we have taken this approach in the hope of getting direct information on the multi-layer sectoral network interdependencies and on how the risk can pass from one to the other. The failure of one subject in the financial network generates a chain reaction through interconnections and causes shocks and therefore a default risk. This risk is understood as the incapability of one of the participants to perform their obligations, or at least to accomplish them properly, which leads to the interruption in the obligation payments of other participants.

One of the most commonly used contagion propagation models corresponds to the celebrated Susceptible-Infected-Susceptible (SIS). In a SIS model, individuals that are cured do not develop permanent immunity, but are again susceptible to the "disease". Similarly, companies that manage to escape default by overcoming high economic stress can fall into trouble again later on. Additionally, SIS model provides valuable insights to understand how different situations may affect the outcome of the contagion process, e.g. what the most efficient technique is for isolating a limited number of companies in a given financial network to minimize the risk of observing an avalanche. Epidemic modeling is still the main application of SIS-like approaches, and the main driver behind the development and refinement of this framework through time. However, the contagion analogy has been applied in different contexts and in particular in those where it is important to consider the spatial and social structure of systems. Some examples are adoption of fads and innovations [9], propagation of news and rumors [10] and information diffusion [11]. These are phenomena for which the state of the agent is affected by the interaction with its neighbors. In the financial context there is a strong causal relation between the financial and economical state of a company's clients and how this influences its economical wellbeing [12]. This dynamics resembles a Hawkes stochastic process [13], where one event, under certain circumstances, is able to generate a new set events allowing the diffusion of a given phenomena [14]. Under this hypothesis, epidemic modeling can shed light on how systemic risk propagates through financial networks. Besides the contagion analogy, there are other similarities between the transmission of diseases and the transmission of financial distress in financial networks. For example, both are branching processes where one event produces others. But we can also observe some differences. For instance, disease transmission is usually studied as a continuous phenomena whereas financial distress is studied in a discrete time scale. Also, there are different levels of homogeneity in both cases, usually financial networks are more heterogeneous than the population networks used in disease spreading research.

In this paper we provide a mechanistic model to assess the impact of a particular diffusion process of default on financial networks. To this end, we take as basis a probabilistic computational framework named microscopic Markov chain approach (MMCA) to compute the probability of the states of individual agents in contagion processes in complex networks [15-17], and adapt its formulation to the understanding of the default propagation in financial networks. Further, we analyze the behavior of our proposed model using real data from the anonymized database of BBVA from December 2015 to December 2016, covering around 140,000 public and private Spanish firms. We set default labels to 0 or 1 
based on this data, depending on whether a given company was or not in default at the beginning of the considered period. By means of this data we have access to the real network of interactions and to the initial condition for the dynamics of the default endogenous propagation.

This paper is organized as follows. First, we review previous work on default propagation in financial networks. Next, in Sect. 3, we propose a contagion model adapting the well-known SIS Model. Section 4 provides a complete description of the data used, and its topological analysis. Section 5 includes a set of experiments to study the main characteristics of default propagation in each inter-connected sector. Later, we examine the implications of using our default propagation model in Sect. 6. Finally, Sect. 7 provides some conclusions and future work.

\section{Related work}

The use of networks in economy and finance has a long tradition. Initial works were conceptual, like [18], where the networks were proposed as tools to represent the interactions (as links) between economic agents playing the role of nodes. When data started to become available, the popularization of complex networks brought a change of paradigm, leading to several advances in the field.

For example, the properties of the economic interchange networks between countries were studied in [19]. Also, the network formed by companies holding shares of other companies was studied for the Milan, New York and NASDAQ stock exchange markets in [20]. Interestingly, these networks show a scale-free nature, which implies that investors having a large number of connections are not uncommon. Explanations for this have been searched in the network dynamics properties mixed with a "rich-gets-richer" effect by means of different approaches [21, 22]. More recent models have been also proposed in $[23,24]$, assuming different hypothesis. A complete review of empirical economic network models can be found at [25]. Beyond the distribution of connections, other characteristics such as the level of clustering have been studied [26]. Despite all these works, there are still numerous open challenges when it comes to fully understanding the structure and dynamics of economic and financial networks [1, 27, 28].

The reason why these networks attract so much attention is that, besides economic interchanges, financial risk also propagates through them [24, 29-32]. Their stability becomes thus an important question [33]. Furthermore, risk and economic distress, and even default in a second stage, can occur in cascades leading to serious systemic instabilities [32, 34]. Therefore, the resilience of the networks to contagion, as well as the circumstances under which it becomes systemic has been analyzed in many works [35-37]. Following this research line, a method called debtrank was introduced to find nodes in financial networks that can induce large cascades when perturbed [3]. This method allows to search for measures to mitigate risk propagation [38]. In the special case of networks where the nodes are banks and the links represent holding of different types of obligations, the complexity of the products traded such as derivatives [39] and the feedback-loops between solvency perception and stock and obligation values [40] can play an important role in economic distress propagation. Many of these previous works have been focused on banking [41, 42], where the risk propagation is related to the stress tests performed by central banks. These kind of models resorts on ad-hoc mathematical models for financial institutions. However, as it has been seen in the last crisis, the risk can spill out of the banking system to enter other economic sectors. This is why it is of high relevance to consider risk 
propagation in more general economic networks, including different sectors and different types of nodes, ranging from large holdings to small companies or even the final individual consumers. This is precisely the direction that we take in the present work where we use general contagion model to evaluate the default spreading in a highly heterogeneous network.

\section{Default contagion model}

Inspired by the Microscopic Markov Chain Approach (MMCA) designed for epidemic spreading, first we propose an adaptation of the framework for modeling the default cascades observed in the transactions between different companies in real financial networks. Then we introduce some measures to dynamically analyze the default contagion process and its functional relations with any sectoral financial network.

\subsection{MMCA model for default contagion}

The original MMCA model was designed to cope with the propagation of epidemics [15], where the states of the agents (nodes) forming the network of contacts where binary, namely, susceptible or infected. In well-mixed populations, the differential equations governing the number of susceptible (S) and infected (I) individuals are

$$
\begin{aligned}
& \frac{d S}{d t}=-\tilde{\beta} S \frac{I}{N}+\tilde{\mu} I, \\
& \frac{d I}{d t}=\tilde{\beta} S \frac{I}{N}-\tilde{\mu} I,
\end{aligned}
$$

where $N=S(t)+I(t)$ is the (constant) size of the population. The term $I / N$ accounts for the probability of contacting an infected individual in a well-mixed population of size $N$, $\tilde{\beta}$ is the infectivity rate (probability per unit time) for each contact, and $\tilde{\mu}$ is the rate at which one infected individual recovers. Their corresponding differential equations are

$$
\begin{aligned}
& S(t+\Delta t)=S(t)\left(1-\tilde{\beta} \Delta t \frac{I(t)}{N}\right)+\tilde{\mu} \Delta t I(t), \\
& I(t+\Delta t)=I(t)\left(1-\tilde{\mu} \Delta t+\tilde{\beta} \Delta t \frac{I(t)}{N} S(t)\right),
\end{aligned}
$$

or equivalently

$$
I(t+\Delta t)=I(t)-\tilde{\mu} \Delta t I(t)+\tilde{\beta} \Delta t \frac{I(t)}{N}[N-I(t)] .
$$

Defining $\rho(t)=I(t) / N$ as the fraction of infected individuals in the population, Eq. (3) is written as

$$
\rho(t+\Delta t)=\rho(t)-\tilde{\mu} \Delta t \rho(t)+\tilde{\beta} \Delta t \rho(t)[1-\rho(t)] .
$$

Note that this discrete equation can be mapped to networks, and in a microscopic approximation, the density of infected individuals will correspond to the individual probability of being infected.

In the case of our economic networks, the state of the different agents corresponds to their liquidity in time according to their initial state and economical activity. To keep 
things as simple and realistic as possible, we assume a discrete-time version of the SIS model in networks, and adopt its terminology, defining $\mu=\tilde{\mu} \Delta t$ as the probability of default recovery, and $\beta=\tilde{\beta} \Delta t$ as the probability of default contagion. In this setting, $\mu$ reflects the average recovery time a company needs to overcome a default process, whereas $\beta$ reveals the average number of interactions with an infected company required to be defaulted. Finally, the network of contacts, in this case, is represented as a weighted matrix, with matrix elements $w i j$ accounting for the total sum of money transfers from node $i$ to node $j$ in 12 months (from a fixed date). The equation governing the default system is then described as:

$$
p_{i}(t+1)=\left(1-q_{i}(t)\right)\left(1-p_{i}(t)\right)+(1-\mu) p_{i}(t)+\mu\left(1-q_{i}(t)\right) p_{i}(t)
$$

where $p_{i}(t)$ is the probability of a node to be in default; $q_{i}=\prod_{j=1}^{N}\left(1-\beta w_{j i} p_{j}(t)\right)$ the probability that a given node $i$ is not infected by any of its neighbors. The right hand side of Eq. (5) is explained as follows: $\left(1-q_{i}(t)\right)\left(1-p_{i}(t)\right)$ is the probability that a given node $i$ is susceptible of entering into default $\left(1-p_{i}(t)\right)$ and it is infected, $\left(1-q_{i}(t)\right)$, by at least one of its neighbors in default. The term $(1-\mu) p_{i}(t)$ is the probability that a node $i$ in default does not recover $(1-\mu)$. Finally, the term $\mu\left(1-q_{i}(t)\right) p_{i}(t)$ corresponds to the probability that a given node $i$ recovers from default but is reinfected by at least one of its neighbors already in default $\left(1-q_{i}(t)\right)$.

According to the European Central Bank definition for risk classification [43], the susceptible state would correspond to a company which is in step 3 (default). In this step, the credit quality of the company is considered equivalent to a probability of default of between $0.10 \%$ and $0.40 \%$ over a one-year horizon. Therefore, after a given period of time (12-18 months), which depends on its revenue, it can go through the step 2 (cure) and finally come back to step 1 (normal) if it proves to have a good payment behavior.

By using this model, we computationally analyze the behavior of default contagion processes in a real topology created by the interactions among different companies. Additionally, we want to understand the main properties of default propagation and the emergent clusters containing defaulted companies in the full system. Moreover, having a welldefined sector distribution and their annual revenue, see Fig. 1, we can elucidate if default

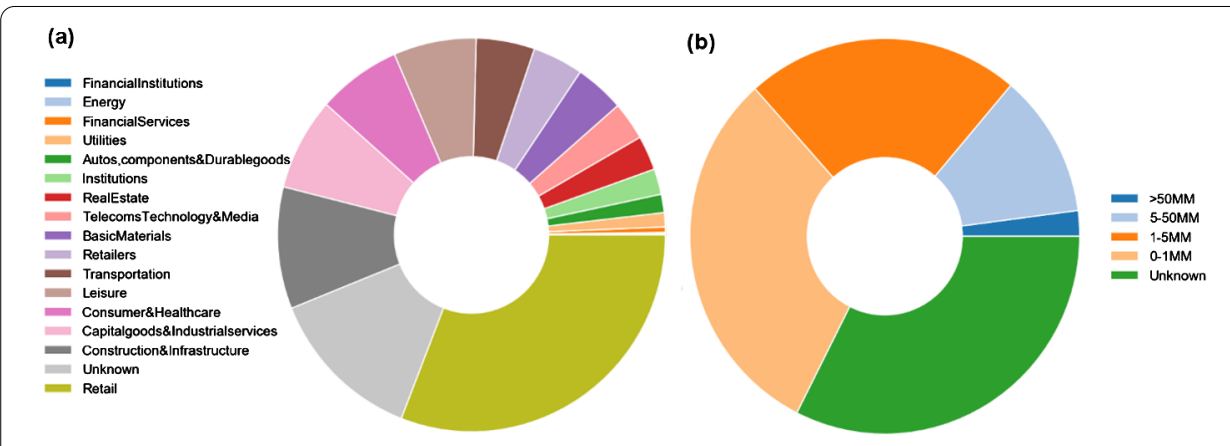

Figure 1 Sector and annual revenue distribution of the customer-supplier network. Distribution of the customer-supplier network by sector and annual revenue. Firms can be classified in 17 sectors according to their NACE code (NACE (Nomenclature of Economic Activities) code is the European statistical classification of economic activities) and main economic activity. Besides, firms can be also classified according to their annual revenue: micro-SME's (less than 1MM EUR, small (1-5MM EUR), SME's (5-50MM EUR) and large (more than $50 M M$ EUR) 
cascades depend on sectors and their economical states. Our objective here is not to build an accurate description of the default process, since for this purpose our model should be more complex and realistic, but to explore the role that the network structure plays on the default propagation process with varying economic scenarios.

\subsection{Dynamical analysis of default contagion}

To analyze the potential for default propagation in a financial network using the defined contagion model, we define a default probability density $\rho$ as

$$
\rho=\frac{\sum_{i=1}^{N} p_{i}^{\mathrm{ss}}}{N}
$$

where $N$ is the number of companies and $p_{i}^{\text {ss }}$ stands for the default probability of company $i$ at the model's stationary state. Note that since we are considering a SIS modeling framework, by construction, the dynamics will always reach a steady state. As shown in Sect. 3.1, the default probability density depends on the default infection rate $\beta$ and the recovery rate $\mu$. For obtaining $\rho$, we monitor $p_{i}(t)$ as a function of time in a discrete manner, until the contagion model reaches the steady state. Note that time represents the iterations of the MMCA recursive model. To do so, we set $p_{i}(0)$ to the real company default label. For understanding the system dynamics, without loss of generality, we can apply the classic MMCA framework where the parameter, $\beta_{i}$, the contagion infectivity per node, is constant and equal for all companies in the network.

However, to make this model more realistic we introduce a variation to this setting using default recovery probability $\mu$ dependent on the relative in-degree of each company $i$. A particular $\mu_{i}$ for a company $i$ is therefore defined as

$$
\mu_{i}=\frac{k_{i}}{\max _{j=1}^{N}\left(k_{j}\right)}
$$

where $\max _{j=1}^{N}\left(k_{j}\right)$ is the maximum in-degree in the network. The intuition behind this heterogeneity in the recovery parameter $\mu_{i}$ is that companies having large number of customers will recover faster than those whose market risk is concentrated in a few customers. Note that this is just one of the possible variations of our default contagion framework. For instance, the generalization of the heterogeneous $\mu$ to other company characteristics/features as balance sheet information or any other individual attribute is straightforward. Moreover, not only the recovery rate but also the infection probability $\beta$ can be defined as company dependent, and/or even depend on group of companies such as economical sector $(C)$. So, Eq. (5) can be generalized as

$$
\left\{\begin{array}{l}
p_{i}(t+1)=\left(1-q_{i}(t)\right)\left(1-p_{i}(t)\right)+\left(1-\mu_{i}\right) p_{i}(t)+\mu_{i}\left(1-q_{i}(t)\right) p_{i}(t), \\
\left.q_{i}=\prod_{j=1}^{N}(1-\beta) w_{j i} p_{j}(t)\right) .
\end{array}\right.
$$

Accordingly, the proposed modification, Eq. (7), to obtain a in-degree dependent $\mu$ is one of the simplest approaches, since it only varies a parameter using a topological characteristic such as the relative in-degree. 


\subsection{Dynamical properties of the network: onset slope and sensitivity}

Naturally, the observed dynamical behavior is the result of an interplay between the MMCA framework and the network topology. To understand the reasons behind the different sectors' response to default propagation, we will characterize the dynamical behavior of each sector by the onset threshold $\mathcal{R}_{0}$ and the sensitivity $S$ to the initial set of defaulted companies. Both metrics are descriptors of the expected behavior in the steady state regime. The onset slope is measured by estimating numerically the critical $\beta_{c}(s)$ at which the first default cases start to appear in sector $C$. Practically, fixing $\mu$, the parameter $\beta$ is increased until the number of default cases in the sector in the stationary state goes over $1 \%$ of the real data defaults found in the sector, marking $\beta_{c}(s)$. All these calculations are done in the stationary state of the system. When $\beta_{c}(s)$ is plotted versus $\mu$, one finds a noisy linear increase and, therefore, we define $\mathcal{R}_{0}$ as the slope of the linear fit.

This property reveals the spreading capacity of the infectious process in each sector. Larger values imply that when the life times of defaults in the companies of the sector become shorter, one needs higher infectivity to overcome the threshold. Sectors with larger $\mathcal{R}_{0}$ should be more resilient to general default. From the moment they start to show significant default, other sectors with lower $\mathcal{R}_{0}$ may be in very bad shape already. Furthermore, given a certain set of parameters, an isolated default event in one of the sectors with larger $\mathcal{R}_{0}$ can trigger an avalanche of default on weaker sectors, for which the conditions are favorable for contagion. In this sense, the $\mathcal{R}_{0}$ value of a sector is also related to the capacity of the sector to spread default.

Regarding the sector sensitivity to default propagation, this dynamical property measures the rate of change of $\rho(\beta, \mu)$ at the transition point (which is normally known as $\beta$ cut-off). Computing sensitivity involves fitting a linear regression to the model response and using its standardized regression coefficients as direct measures of sensitivity. Therefore this metric describes how susceptible a sector is to default, quite the opposite to $\mathcal{R}_{0}$, which characterizes how a sector affects the system. The relationship between these two dynamical descriptors and the network structure will be explored next. As mentioned before, these are defined at the steady state, but it is also important to understand how dynamics evolve in the transient regime. This analysis will be carried out by synthetically concentrating defaulted companies (in specific proportions) in the different sectors and exploring pair-wise sectoral interactions at the initial steps of the simulation.

\subsection{How do sectoral properties of the nodes affect network dynamics?}

Since economic crisis often start in a given sector and later expand to others (see for example the 2000 energy crisis and the 2008 financial crisis), we are also interested in exploring the dependence of default propagation on attributes related to each sector, such as sectoral default probability density and sectoral inter-connectivity. In particular, to study the dependence on the sectoral default probability density we rewrite Eq. (6) for a each sector $(C)$ as:

$$
\rho_{C}=\frac{\sum_{i \in C} p_{i}^{\mathrm{ss}}}{N_{C}}
$$


We also propose a metric of in-sectoral inter-connectivity $I_{\text {in }}$ that measures how well, on average, a sector is connected to other sectors by incoming links:

$$
I_{\text {in }}=\frac{\sum_{i, j}\left(w_{i j}-\frac{s_{i}^{\mathrm{in}}}{17}\right)^{2}}{N_{C}},
$$

where $N_{C}$ is the number of companies a certain sector $C$ has, $i \in C$, $w_{i j}$ are incoming links of $i(j \rightarrow i)$ and $s_{i}^{\text {in }}$ is the company $i$ in-strength. In short, the in-sector inter-connectivity measures the mean square error with respect to a hypothetical equally distributed situation where we have a node with incoming weights from all $C$ sectors with equal probability

$\left(\frac{s_{i}^{\text {in }}}{C}\right)$. So, the larger the value, the more heterogeneously connected the sector is to other sectors by incoming links.

\section{Topological analysis of the client-supplier network}

Now we describe the sectoral financial network used in the experiments carried out in this work. To do so, we first provide all the details about network construction. Second, we report commonly used network statistical descriptors.

\subsection{Network construction}

Customer-supplier relationships highly depend on economical sectors and the financial situation of the companies involved. To properly model this situation with real data we gathered anonymized quarterly data from the official customer-supplier third party payment declarations collected by the BBVA risk management department. This declaration is used as a mechanism to avoid fraud in company VAT declarations. here, Spanish firms (our nodes) inform about their supplier payments and customer earnings. For each available company, we extracted its operating revenue and financial statement attributes: sector and default status. Collected data covers from December 2015 to December 2016. Default labels at the initial step were set to 0 or 1 depending on whether a company was in default or not at the beginning of this period. By using customer-supplier relationships, and after removing self-loops, a directed and weighted network with 142,477 nodes and 255,509 edges was obtained. Direction of edges follows the path of money injection (from the customer to the supplier). All edge weights (total money transferred) were aggregated annually and normalized by its source node out-strength. Note that, both BBVA customers and non-customers were included in a percentage of $63 \%$ and $37 \%$, respectively. Therefore, the network contains an important percentage of missing values.

As illustrated in Fig. 1, most of the companies included in the network are micro-SME's and small companies, with an annual revenue smaller than 5 million euros (more than $80 \%$ of the informed values). Besides, the most common categories are retail (shops), followed by construction \& industrial companies. It is important to mention that leisure and consumer \& healthcare are also important sectors in the network. Although nodes belonging to energy and financial institutions are only a few, they account for $50 \%$ of the network's out-strength, whereas other sectors such as retail are relatively abundant in the network (30\% of the nodes) but only account for $3 \%$ of the total out-strength. To model the probability of contagion, we normalized the edge weights by the out-strength of the source node (customer).As a result, Energy and Financial Institutions account for the $11 \%$ of the normalized out-strength in the network, and Retail for the 5\% (20\% and 7\% of the corresponding global in-strength). See Table 1 for more details. 
Table 1 Summary of network topological measures by sector where $\bar{k}_{\text {in }}$ and $\bar{k}_{\text {out }}$ stand for the average in-degree and out-degree, respectively

\begin{tabular}{lrrrllc}
\hline Sector & Size (\%) & \multicolumn{1}{c}{$\bar{k}_{\text {in }}$} & \multicolumn{1}{c}{$\bar{k}_{\text {out }}$} & Default (\%) & Rank hub & Rank auth \\
\hline Financial institutions & 0.046 & 39.613 & 45.529 & 3.650 & 17 & 1 \\
Energy & 0.083 & 12.844 & 8.666 & 1.111 & 14 & 2 \\
Financial services & 1.165 & 6.300 & 20.265 & 0.786 & 13 & 3 \\
Utilities & 1.529 & 5.589 & 5.903 & 1.264 & 11 & 4 \\
Telecoms technology \& media & 3.299 & 5.960 & 5.194 & 1.776 & 2 & 5 \\
Basic materials & 2.745 & 5.789 & 5.350 & 2.782 & 6 & 6 \\
Transportation & 4.064 & 5.411 & 4.336 & 1.868 & 1 & 7 \\
Retail & 23.593 & 3.973 & 3.233 & 1.217 & 12 & 8 \\
Retailers & 4.273 & 5.001 & 3.613 & 1.885 & 5 & 9 \\
Capital goods \& industrial services & 8.689 & 4.528 & 3.098 & 1.866 & 9 & 10 \\
Autos, components \& durable goods & 1.470 & 4.454 & 2.991 & 1.786 & 10 & 11 \\
Consumer \& healthcare & 7.055 & 3.259 & 3.770 & 1.539 & 8 & 12 \\
Construction \& infrastructure & 8.907 & 3.067 & 3.270 & 3.071 & 3 & 13 \\
Unknown & 10.159 & 0.930 & 1.413 & 1.942 & 15 & 14 \\
Real rstate & 6.843 & 1.517 & 1.844 & 3.603 & 7 & 15 \\
Leisure & 12.861 & 2.509 & 2.512 & 1.511 & 4 & 16 \\
Institutions & 3.219 & 5.547 & 10.764 & 0.535 & 16 & 17 \\
\hline
\end{tabular}
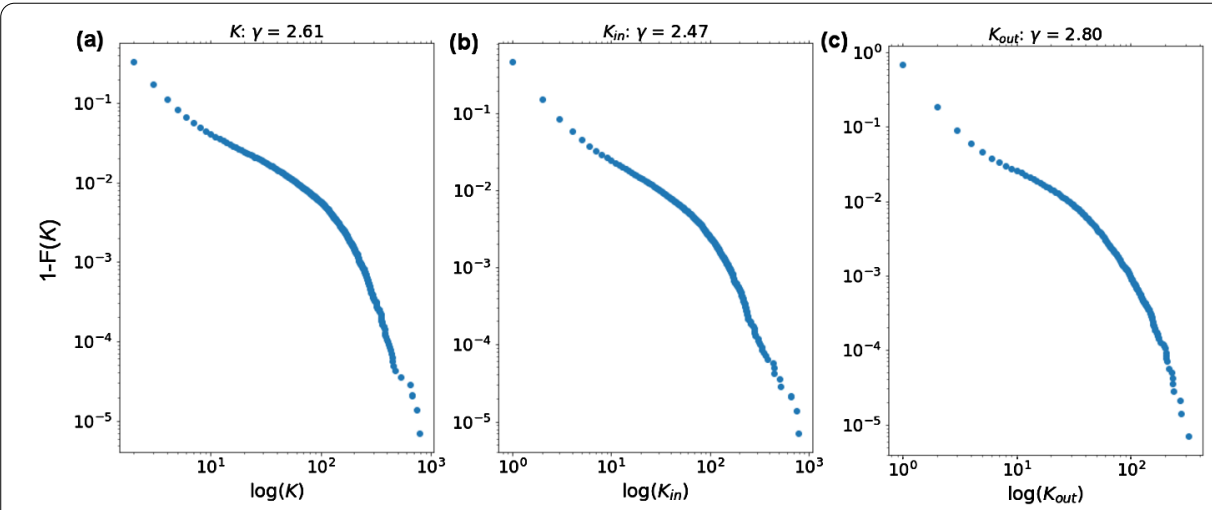

Figure 2 Degree distribution of the customer-supplier network. Client-supplier network degree distribution for nodes (a) degree, (b) in-degree and (c) out-degree. The networks are highly heterogeneous and heavy tailed

\subsection{Statistical descriptors}

The first point we address is the degree distribution of the network. The degree distributions of the real network analyzed (total, in- and out-degree) presents a heavy-tail but does not fit to a power-law. This heavy-tail is relevant for the sake of the analysis given that it clearly indicates the existence of hubs. The complementary cumulative distribution functions are displayed in Fig. 2. Table 1 also sorts business sectors according to their average hub and authority score [44]. The main hub in the network is the transportation sector, followed by telecoms, Technology \& media and construction \& infrastructure sectors. The main authority is related to financial institutions, followed by energy and financial services. Hubs and authorities agree with the expected economic behavior. Hub sectors such as transportation, Construction \& Infrastructure and telecoms, Technology \& media, which require energy resources to produce goods, transport them, or even to run IT services, therefore those sectors have important connections to authorities such as energy. Note also sectors such as financial institutions and services (credit cards, insurances) arising as important authorities. This is a direct result of the financial needs that many the 
companies have to operate in Spain. This need is mostly because the average number of days required to collect invoiced amounts from customers is quite high in Spain, around 90 days.

\section{Experimental results}

We will study next three different default propagation scenarios. The first one corresponds to the classical MMCA model where all nodes share the same recovery rate. In the second one we use the heterogeneous recovery rate measure introduced in Sect. 3.2. Besides, to increase our knowledge about the role of each sector in the default propagation process, we synthetically simulate default problems in each sector to analyze the different spreading speed in the transient state. Finally, we validate our findings comparing achieved results with a null model built by rewiring the edges of our network.

\subsection{Default incidences for homogeneous recovery rate}

We first explore the system response to the default dynamics using the baseline model. Figure 3 shows the default probability density at the steady state versus the default infection rate $\beta$ for a default recovery rate of $\mu=0.01$. For such a small recovery rate the system behaves almost like a Susceptible-Infected model, still there is a non-zero transition point from a low default rate to a system-wide default regime. As shown, for high infectious rates, the system default density is close to 0.20 . This means that on average companies in the customer-supplier network, have a 0.20 probability of being in default for the set of parameters used.

Figure 4 shows $\rho(\beta, \mu)$ for each economical sector. Clearly, not all sectors behave in the same way regarding default dynamics. Broadly speaking, economical sectors can be grouped in three blocks given its response to default propagation. On one hand, (public) Institutions, Leisure and unknown sector reference show a low propensity to default propagation, where the sector default probability density range from approximately 0.04 to 0.10 for high infectious rate $\beta$. On the other hand, Financial Institutions and Energy evince a high propensity to default contagion, with $\rho$ reaching almost 0.50 . In other words, on average, each company of these two sectors has a 0.50 probability of being in default for

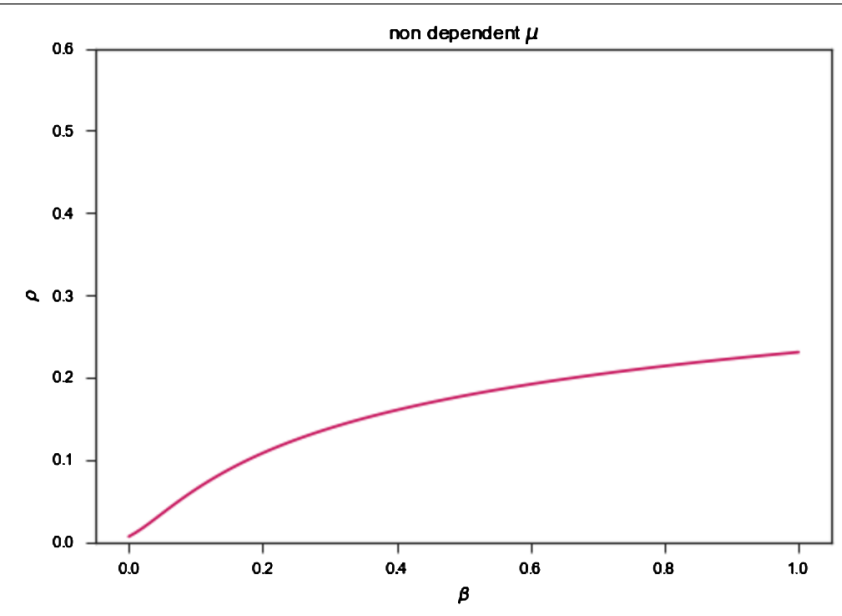

Figure 3 Recovery rate dependence of the fraction of infected nodes. $\rho(\beta, \mu)$ for the system with the recovery rate $\mu$ for all network nodes (homogeneous) 


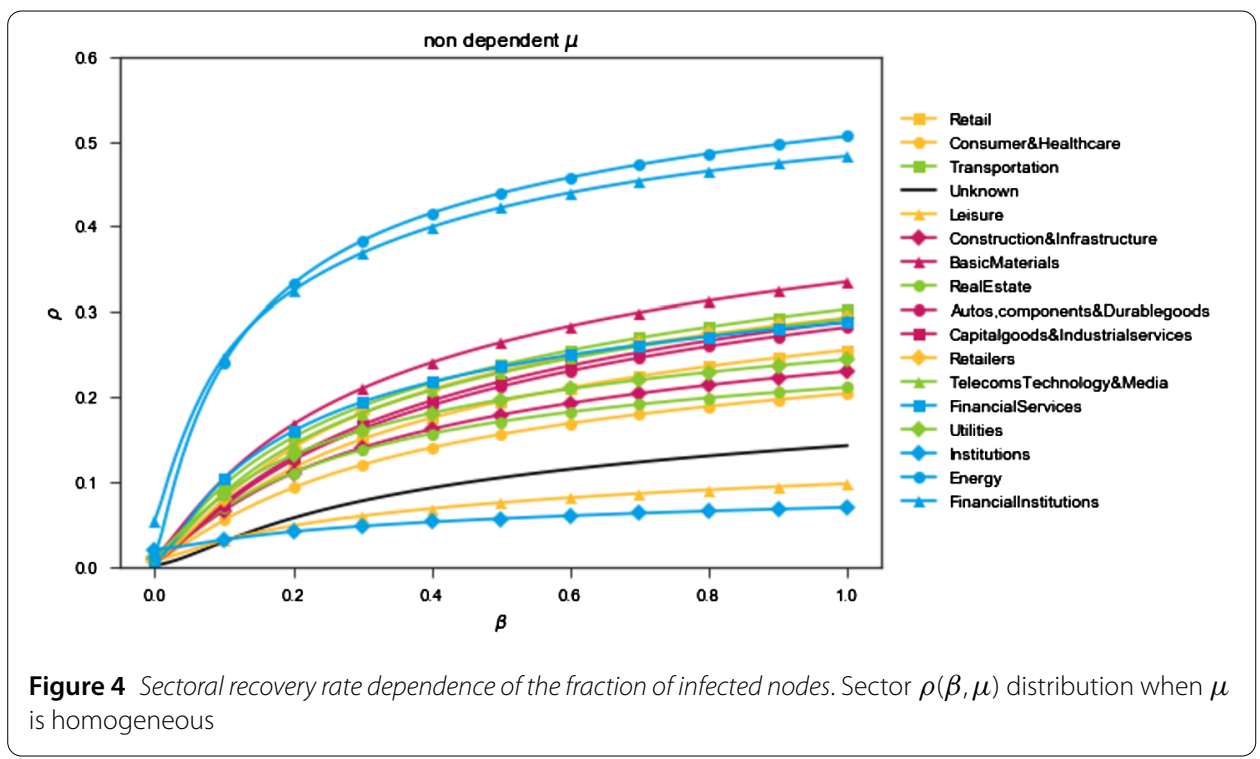

extreme parameter conditions. In-between, we find the rest of the sectors with density variations ranging from 0.15 to almost 0.30 . Interestingly, the exposure of the economical sectors is quite different one to another. This result, may allow current risk assessment models (e.g. Generalized Linear Models, GLM) to include a quantification of sectoral risk and rank accordingly. In the following sections, we will assess the effect of the recovery rate variation and explore the reason behind the different sector response to default dynamics.

\subsection{Impact of customer diversification on default incidence}

In addition, we have compared the customer diversification variation model with a baseline model having constant default recovery rate $\mu$. We simulate the latter with $\mu$ equal to the mean of $\mu_{i}$ for the whole network, specifically with $\mu=0.005$. Consequently, the homogeneous $\mu$ (baseline model) used is 0.01 . In our data, initial conditions for the number of companies in default at $t_{0}$ are more concentrated in Financial Institutions, $15 \%$ (relative to the sector) and the rest varies with a default rate between $6 \%$ and $1 \%$. However, the MMCA modeling framework does not depend on the initial conditions when the steady state is reached.

Figure 5 depicts $\rho(\beta, \mu)$ distribution for each sector using a heterogeneous $\mu_{i}$ dependent of the companies customer diversification. Default density $\rho$ increases when it is compared to the homogeneous $\mu$ scenario for all sectors. This means that the inclusion of recovery heterogeneity make sectors more prone to default. Nevertheless, this effect is likely related with the Spanish financial network structure, where medium and small companies with low diversification are predominant in the system (see Fig. 1 ). This fact produces that most nodes have small $\mu$ values (close to zero), whilst very few companies present $\mu$ values close to 1 . However, there are some differences for a few sectors. We will explore the reason behind the observed dynamic behavior and its connection to the network structure in the following section.

\subsubsection{Sector structure-function relationship}

Table 2 summarizes metrics related to the network dynamics and topology for all sectors. The onset slope $\mathcal{R}_{0}$ and the rank according to the sensitivity value for the baseline 


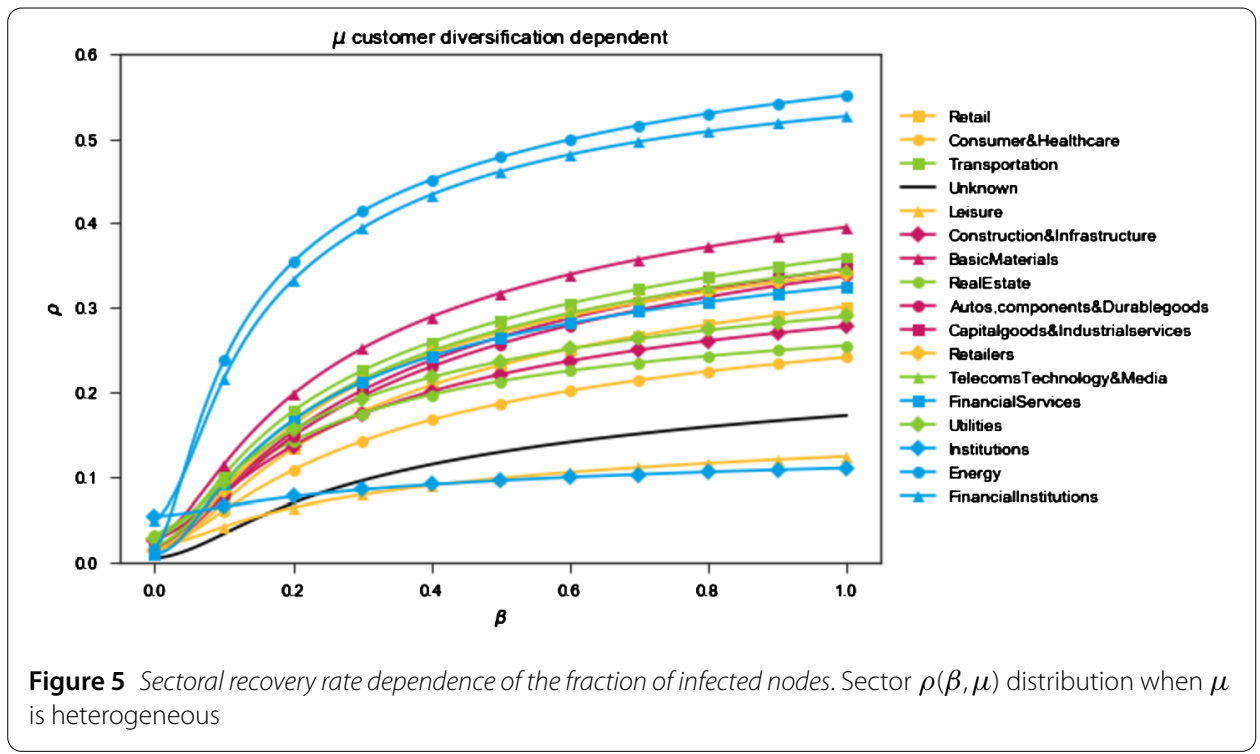

Table 2 Summary of network measures influencing dynamic default contagion by sector

\begin{tabular}{lcrccc}
\hline Sector & $\gamma$ & \multicolumn{1}{c}{$l_{\text {in }}$} & $\mathcal{R}_{0}$ & $S_{\text {het }}^{\text {rank }}$ & $S_{\text {hom }}^{\text {rank }}$ \\
\hline Energy & 1.33 & 13.44 & 1.74 & 1 & 1 \\
Financial institutions & 1.27 & 2.39 & 3.81 & 2 & 2 \\
Basic materials & 1.58 & 41.07 & 2.89 & 3 & 5 \\
Financial services & 1.36 & 1.66 & 2.22 & 4 & 3 \\
Transportation & 1.44 & 16.27 & 2.33 & 5 & 4 \\
Telecoms, technology \& media & 1.44 & 16.99 & 2.68 & 6 & 6 \\
Retailers & 1.54 & 28.18 & 2.70 & 7 & 8 \\
Capital, goods \& industrial services & 1.47 & 9.51 & 2.93 & 8 & 9 \\
Utilities & 1.44 & 7.15 & 3.43 & 9 & 7 \\
Autos, components \& durable goods & 1.52 & 21.24 & 2.82 & 10 & 10 \\
Retail & 1.58 & 29.80 & 2.73 & 11 & 11 \\
Real estate & 1.40 & 1.86 & 4.74 & 12 & 12 \\
Construction \& infrastructure & 1.44 & 17.01 & 4.25 & 13 & 13 \\
Consumer \& health care & 1.55 & 32.06 & 4.10 & 14 & 14 \\
Unknown & 1.59 & 5.16 & 3.69 & 15 & 15 \\
Leisure & 1.35 & 7.71 & 7.56 & 16 & 16 \\
Institutions & 1.54 & 5.65 & - & 17 & 17 \\
\hline
\end{tabular}

model $S_{\text {hom }}^{\text {rank }}$ and for the customer diversification variation $S_{\text {het }}^{\text {rank }}$ are shown. Worth noting, we discarded the $\mathcal{R}_{0}$ value corresponding to Institutions (a sector including governmental institutions, religious organizations and others public institutions) because there were very few points, and as a consequence the regression slope is not statistically robust. In general, it is observed that there are slight variations in the sensitivity ranking but, as seen on Figs. 4 and 5, the customer diversification has not modified how most of the sectors are affected by the default dynamics. Also, there is quite a strong inverse relationship between the onset slope and the sensitivity, indicating that sectors which are susceptible to be affected are not those prone to propagate the default throughout the system. Main changes are observed in the basic materials and utility sectors. For example, default sensitivity of basic material companies which have a strong inter-sectoral inter-connectivity is reduced, showing that these companies have a very diverse customers, therefore making them more resistant to default spreading. Here it is important to highlight that the network only contains business to business relations, therefore sectors whose customers 
are mostly individuals clients have lower probability to spread a default process. Besides, sectors having a larger number of authorities (nodes highly connected to hubs) exhibit a larger centrality making them more suitable to spread default problems.

We still lack an explanation of the observed behavior given the topological characteristics of each sector. To do so, we computed the slope coefficient of the inverse cumulative probability distribution of the companies in-strength for each sector $(\gamma)$. The in-strength is defined as the sum of the incoming normalized weights for each company, meaning that the higher the value for a company the more probable that the default dynamics will affect it. As in most complex systems, this probability distribution is heavy-tailed signaling a Pareto like distribution where the slope coefficient can be computed. A smaller $\gamma$ value signals that the sector is more probable to contain well connected companies (sector hubs). We observe that Energy and Financial Institutions are the most susceptible ( $\left.S^{\text {rank }}\right)$ sectors, and coincide with lower values of $\gamma$ (higher probability of sector hubs). The contrary happens to Institutions, and unknown sector reference. This highlights the fact that hub structures play an important role in the dynamics, and in the extent that a sector is affected by it. Clearly, leisure does not follow this explanation because it has a middlerange $\gamma$ value. This could be due to the fact that it has $76 \%$ of its companies with zero in-strength. However, it is naive to think that only this structural property can explain the sector response to default dynamics. In Table 2, we can observe how less susceptible sectors are more equally inter-connected to other sectors (larger value of $I_{\text {in }}$ ). In practice, this causes the default spreading to be less likely to find a high probability path to these sectors because their incoming weights are less concentrated. As before, economical sectors can be grouped in three blocks given its capacity to interact and affect other sectors. On one hand, energy, financial institutions, financial services, transportation, telecommunications and retailers sectors are largely affected by the others due to their large sensitivity. Besides, these sectors are highly inter-connected with all the other sectors since their activity is traversal to all sectors and firms, therefore this high degree connectivity allows default to infect them easily. On the other hand, when consider leisure and unknown sectors, we observe that these are not affected by other sectors. For leisure sector, this is a consequence that most of its companies have zero in-strength. Similarly, companies belonging to the unknown sector are not BBVA clients, so their information is quite limited and most of their connections are not included in the network having, both, low in and out degree. In-between, there are sectors, such as (public) institutions and retail, that are stable independent of the perturbed sector. The main characteristic of these sectors is their low in-strength due to their customers not being companies or not having customers at all because they are public entities. In any case, default contagion does not reach these sectors and they kept healthy in all parameters setting.

Independently of the particular economic insights that may arise from these analysis, the methodology proposed in this paper has a greater advantage. It allows to perform experiments in a massive way. These large amount of data has enabled us to study how the time it takes the system to arrive to the steady state (convergence time) depends on a set of parameters such as the initial default rate or the infection rate $\beta$. This may have practical applications. For instance, if we can establish a relationship between simulations convergence time and real time, the risk departments could take advantage of this understanding to estimate the speed of default propagation among sectors. 


\subsection{Synthetic default assessment experiments}

The methodology also allows for another kind of experiments; what we call synthetic default assessment. Previously, we have performed a sensitivity analysis using the real default distribution at the initial step. Now we are going to explore what happens when the initial default nodes are concentrated in a given sector and repeat the analysis for every sector. Previously, we have focused on the stationary state, where dynamic properties such as $S$ and $\mathcal{R}_{0}$ were calculated without any dependence on the initial conditions. Now, we are interested in the transient phase, when the system has not reached the stationary state yet. In this transient phase, we can gain insights on the speed of default propagation among sectors.

To perform this experiment, we have randomly set different initial default values, specifically, $2 \%, 4 \%, 8 \%$ and $25 \%$ in each sector and no default in the remaining nodes (in the real data there is an initial default ratio equal to $1.703 \%$, distributed across the sectors). Although, several experiments were performed, here we only include two of them, the ones described in Figs. 6 and 7. Simulations show that the moment when the system arrives to the stationary state depends on the initial default values, the infection rate $\beta$ and the recovery rate $\mu$. In particular, the larger the recovery rate $\mu$, the faster the system arrives to the stationary state. Note that for initial default values of $25 \%$ (Fig. 7 ) there are no horizontal lines at iteration 20, indicating that the affected sector is still in the transient state, since it depends on the perturbed sector. We also observe that there are difference among sectors. Energy is the sector which first reaches the stationary for all values of the parameters.

We observe that most of the business sectors follow the tendency that the larger the number of sectors they affect, the fewer the number of sectors that at the same time are affecting them. An example can be found in Utilities; for a $25 \%$ perturbation all other sectors except one are affected, while Utilities itself is only affected by another sector (Unknown sector). Note that this is also the case for Transportation, and seems to be a constant throughout the other sectors. This is opposite to what happens to the sectors Energy and Financial Institutions, which is affected rapidly by all the other sectors. Knowing the dependence of the default speed contagion on the business sectors may allow risk assessment models to understand the conditions to react to a sudden perturbation of a sector, or to an event that may indicate the initial stages of a sector crisis.

\subsection{Validation}

To study if the topological properties of the customer-supplier network really affect default contagion, edges were rewired a million times in such a way that nodes kept their out-degree, in-degree and out-strength using the algorithm described in [45]. Doing this, 25 different networks with different topology were created. In Fig. 8, we present the differences of the trimmed mean of $\rho$ as a function of $\beta$ with regard to the original network by sector. Specifically, bloxplots were computed using the formula:

$$
\Delta \rho_{s}=\frac{\left|\sum_{n=P_{5}}^{P_{95}} \frac{\rho_{r, n}}{N_{r, s}}-\sum_{n=P_{5}}^{P_{95}} \frac{\rho_{o, n}}{N_{o, s}}\right|}{\sum_{n=P_{5}}^{P_{95}} \frac{\rho_{o, n}}{N_{o, s}}},
$$

where $P_{i}, \rho_{r}, \rho_{o}$ stand for the $i$ th percentile, node $\rho$ values of the rewired network and $\rho$ values of the original network respectively. Besides, $N_{r, s}$ and $N_{o, s}$ are the number of nodes 


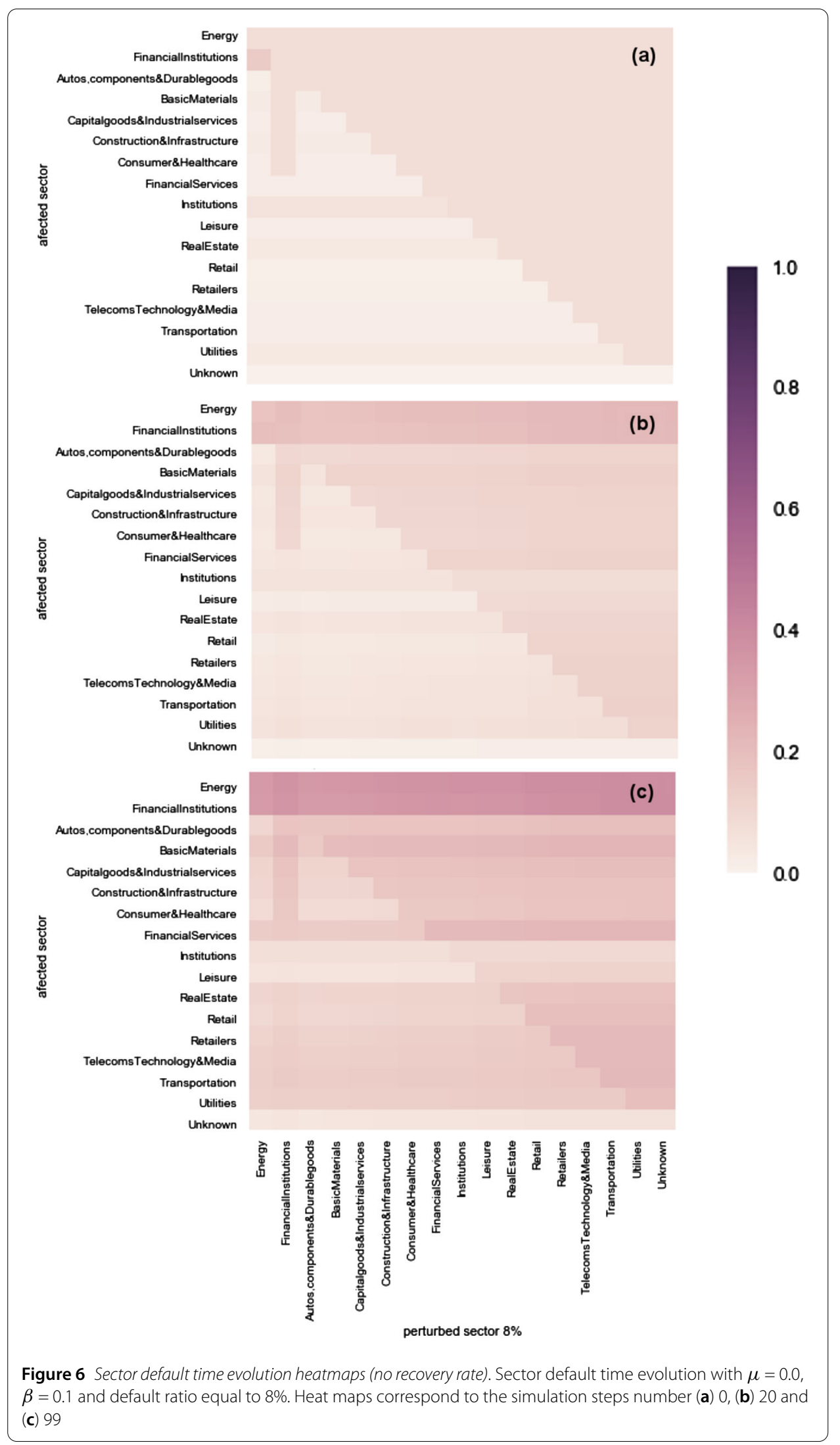




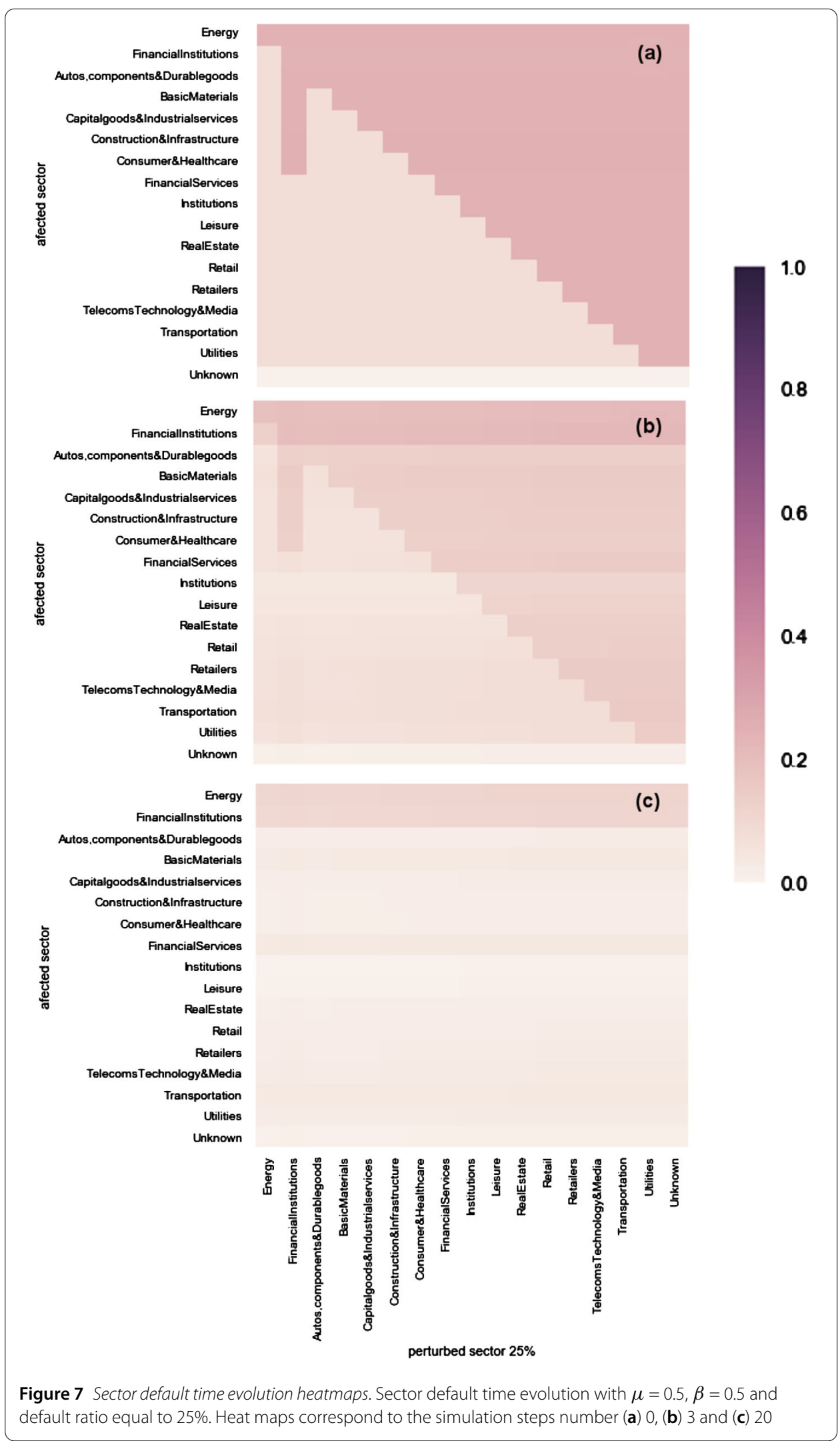




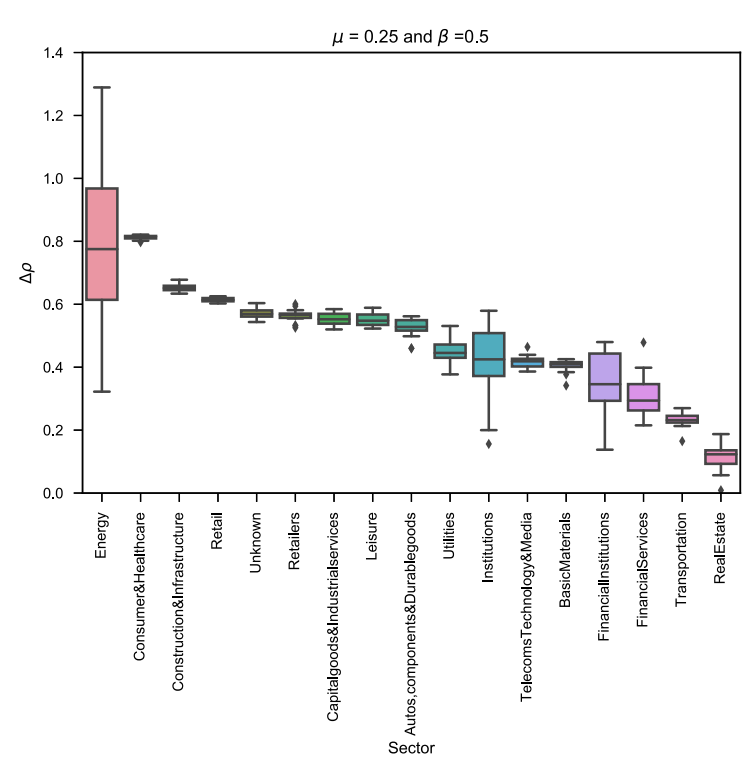

Figure 8 Null model for the sector default distribution. Sector $\Delta \rho$ distribution obtained from rewiring 25 times client-supplier network with $\mu=0.25$ and $\beta=0.5$

within percentiles $P_{5}$ and $P_{95}$ of the rewired and original network respectively. $\Delta \rho$ values of Fig. 8 show significant differences between customer-supplier network and the rewire one. Consequently, this confirms that the customer-supplier network structure plays an important role in all the aforementioned results.

\section{Discussion}

The methodology presented in this work applied to a real customer-supplier network of companies in Spain has allowed to gain new insights on financial data. We confirm quantitatively that business sector is a key component in default contagion, both in the strength and in the velocity of the contagion. Specifically we have found three differentiated blocks of behaviors depending on the business sectors. The ability to quantitatively estimate the size of this effect via topological analysis of the customer-supplier network demonstrated in this work may allow current risk assessment models to include them for future default prediction in a systematic way. Nowadays, this effect is only included from macro-financial data perspective. Therefore, there is a lack of relational information at micro-level. Customer-supplier network relations would cover this gap. We have proven that this dependence happens in at least two different scenarios: when recovery rate is homogeneous and when it is heterogeneous for every company. However, when the recovery rate depends on a topological property such as customer diversification, default contagion increases. This finding may be only a consequence of the structure of the customer-supplier network in Spain. Further studies could be carried out by using other country customer-supplier networks, and by using other dependence hypothesis for the recovery rate. This is where a major advantage of the methodology presented in this work comes: the ability to vary infection and recovery parameters at the micro-scale and study the effects on the dynamical properties of the network. For instance, we can base the model on more economically-wise hypotheses for the recovery rate (or infectious rate) that account for more realistic scenarios, such as the companies' revenue, and answer why some 
sectors do not default although they should, provided the structure of the network. This allows risk experts to study many possible hypothesis. After carrying out a detailed analysis of how different parameters affects default contagion, we have seen that the number of hub companies a business sector includes is essential to estimate its sensitivity to default contagion. Although this is not the only variable to take into account, and results can not be generalized to other customer-supplier networks outside of Spain, we believe this is a first step towards the study of how the topological properties of the companies in the network affect default contagion. From a topological perspective, the results of our model applied to the real data reveal which sectors are more at risk in the propagation of default, which sectors are more resilient to the default avalanches, and what are the expectations for the cascades of default under different stochastic conditions. For that purpose, we have carried out two type of experiments:

- We have analyzed the dynamics of default contagion for different values of the recovery parameter $\mu$, dependent and non-dependent on the node's features. Our methodology allows to tune parameters individually for every company and to carry out experiments for the simulation of future scenarios. In particular, we have studied the impact of the company's customer diversification on default propagation.

A discussion on the connection between topological and dynamical properties is also included (Sect. 5.2.1);

- Our methodology also allows for another kind of experiment described in Sect. 5.3, where we have focused on the dynamics of default propagation at the transient state, and its dependence on the default initial conditions.

\section{Conclusions}

We have proposed a computational model, based on the probabilities of default contagion, to study the default diffusion at individual and aggregated levels. We have performed massive experiments based on this model by varying several parameters such as the initial default rate, the contagion rate $\beta$ and the recovery rate $\mu$. This methodology allows us to vary the parameters at the individual level to account for a more realistic scenarios. Our results show the relationship between dynamical and topological properties for more than 140,000 BBVA firms aggregated at a economic sector level, and also allow us to create a ranking of sectors by sensitivity to default, which can be used in potential applications. For future work, we would like to enrich the network adding different types of payments such as national transfers or direct debits, extending in this way our computational model to a multiplex network, finally we want to enrich model parameters considering for example companies' revenue.

\footnotetext{
Funding

JJR acknowledge partial funding from the Spanish Ministry of Science, Innovation and Universities, the National Agency for Research Funding AEl and FEDER (EU) under the grant PACSS (RTI2018-093732-B-C22) and the Maria de Maeztu program for Units of Excellence in R\&D (MDM-2017-0711). AA acknowledges support by Spanish Ministry of Science, Innovation and Universities (grant PGC2018-094754-B-C21), Generalitat de Catalunya (grant 2017SGR896), Universitat Rovira i Virgili (grant 2017PFRURV-B2-41), ICREA Academia and the James S. McDonnell Foundation (grant 220020325). We acknowledge support of the publication fee by the CSIC Open Access Publication Support Initiative through its Unit of Information Resources for Research (URICI). The funders had no role in study design, data collection and analysis, decision to publish, or preparation of the manuscript.
}

\section{Abbreviations}

BBVA, Banco Bilbao Vizcaya Argentaria, name of the second largest bank in Spain; GLM, Generalized Linear Models; MMCA, Microscopic Markov Chain Approach; SIS, Susceptible-Infected-Susceptible epidemic model; SME, Small and Medium-sized Enterprise; VAT, Value-Added Tax. 
Availability of data and materials

The dataset is not publicly available. It was acquired by BBVA. Any of the authors based at BBVA may be contacted for further details about the dataset.

\section{Competing interests}

The authors declare that they have no competing interests.

\section{Authors' contributions}

Conceptualization: JN, ET, PF, AA, JJR; methodology: AA; formal analysis: AB, JN, PF, ET; data curation: ET; writing (original draft preparation): PF, ET, JN; writing (review and editing): JN, PF, ET, AA, JJR; visualization: JN, $A B, A M, E T$; funding acquisition: JN. All authors read and approved the final manuscript.

\section{Author details}

${ }^{1}$ BBVA Data \& Analytics, Barcelona, Spain. ²Dept. Enginyeria Informàtica i Matemàtiques, Universitat Rovira i Virgili, Tarragona, Spain. ${ }^{3}$ ESADE, Universitat Ramon Llull, Barcelona, Spain. ${ }^{4}$ Instituto de Física Interdisciplinar y Sistemas Complejos IFISC (CSIC-UIB), Palma de Mallorca, Spain.

\section{Publisher's Note}

Springer Nature remains neutral with regard to jurisdictional claims in published maps and institutional affiliations.

Received: 9 July 2018 Accepted: 17 October 2019 Published online: 04 November 2019

\section{References}

1. Schweitzer F, Fagiolo G, Sornette D, Vega-Redondo F, Vespignani A, White DR (2009) Economic networks: the new challenges. Science 325:422-425. https://doi.org/10.1126/science.1173644

2. Haldane AG, May RM (2011) Systemic risk in banking ecosystems. Nature 469:351-355. https://doi.org/10.1038/nature09659

3. Battiston S, Puliga M, Kaushik R, Tasca P, Caldarelli G (2012) DebtRank: too central to fail? Financial networks, the FED and systemic risk. Sci Rep 2:541. https://doi.org/10.1038/srep00541

4. Roukny T, Bersini H, Pirotte H, Caldarelli G, Battiston S (2013) Default cascades in complex networks: topology and systemic risk. Sci Rep 3:2759. https://doi.org/10.1038/srep02759

5. Pastor-Satorras R, Castellano C, Van Mieghem P, Vespignani A (2015) Epidemic processes in complex networks. Rev Mod Phys 87:925-979. https://doi.org/10.1103/RevModPhys.87.925

6. Wu Y (2014) Reputation risk contagion and control of rural banks in China based on epidemic model. Comput Model New Technol 18:229-235

7. Paltalidis N, Gounopoulos D, Kizys R, Koutelidakis Y (2015) Transmission channels of systemic risk and contagion in the European financial network. J Bank Finance 61:S36-S52. https://doi.org/10.1016/j.jbankfin.2015.03.021

8. Kostylenko O, Rodrigues HS, Torres DFM (2019) The spread of a financial virus through Europe and beyond. AIMS Math 4:86. https://doi.org/10.3934/Math.2019.1.86

9. Rogers EM (2010) Diffusion of innovations. Simon \& Schuster, New York

10. Jin F, Dougherty E, Saraf P, Cao Y, Ramakrishnan N (2013) Epidemiological modeling of news and rumors on Twitter In: Proceedings of the 7th workshop on social network mining and analysis, $\mathrm{p} 8$

11. Khelil A, Becker C, Tian J, Rothermel K (2002) An epidemic model for information diffusion in MANETs. In: Proceedings of the 5th ACM international workshop on modeling analysis and simulation of wireless and mobile systems, Atlanta, GA, USA, pp 54-60

12. Mizgier KJ, Wagner SM, Holyst JA (2012) Modeling defaults of companies in multi-stage supply chain networks. Int J Prod Econ 135:14-23

13. Rizoiu M-A, Mishra S, Kong Q, Carman M, Xie L (2018) SIR-Hawkes: linking epidemic models and Hawkes processes to model diffusions in finite populations. In: Proc. of the World Wide Web conference (WWW), pp 419-428

14. Glasserman P, Young HP (2016) Contagion in financial networks. J Econ Lit 54:779-831. https://doi.org/10.1257/jel.20151228

15. Gómez S, Arenas A, Borge-Holthoefer J, Meloni S, Moreno Y (2010) Discrete-time Markov chain approach to contact-based disease spreading in complex networks. Europhys Lett 89:38009. https://doi.org/10.1209/0295-5075/89/38009

16. Gómez S, Gómez-Gardeñes J, Moreno Y, Arenas A (2011) Nonperturbative heterogeneous mean-field approach to epidemic spreading in complex networks. Phys Rev E 84:036105. https://doi.org/10.1103/PhysRevE.84.036105

17. Cai C-R, Wu Z-X, Guan J-Y (2014) Effective degree Markov-chain approach for discrete-time epidemic processes on uncorrelated networks. Phys Rev E 90:052803. https://doi.org/10.1103/PhysRevE.90.052803

18. Callon M (1990) Techno-economic networks and irreversibility. Sociol Rev 38:132-161

19. Serrano MA, Boguñá M (2003) Topology of the world trade web. Phys Rev E 68:015101. https://doi.org/10.1103/PhysRevE.68.015101

20. Garlaschelli D, Battiston S, Castri M, Servedio VDP, Caldarelli G (2004) The scale-free topology of market investments. Phys A, Stat Mech Appl 350:491-499. https://doi.org/10.1016/j.physa.2004.11.040

21. Jackson MO, Watts A (2002) The evolution of social and economic networks. J Econ Theory 106:265-295 https://doi.org/10.1006/jeth.2001.2903

22. Coelho R, Neda Z, Ramasco JJ, Santos MA (2005) A family-network model for wealth distribution in societies. Phys A Stat Mech Appl 353:515-528. https://doi.org/10.1016/j.physa.2005.01.037

23. Gale DM, Kariv S (2007) Financial networks. Am Econ Rev 97:99-103

24. Babus A (2016) The formation of financial networks. Rand J Econ 47:239-272. https://doi.org/10.1111/1756-2171.12126 
25. Kosfeld M (2004) Economic networks in the laboratory: a survey. Rev Network Econom 3:1 https://doi.org/10.2202/1446-9022.1041

26. Onnela J-P, Kaski K, Kertész J (2004) Clustering and information in correlation based financial networks. Eur Phys J B 38:353-362. https://doi.org/10.1140/epjb/e2004-00128-7

27. Battiston S, Glattfelder JB, Garlaschelli D, Lillo F, Caldarelli G (2010) In: Estrada E, Fox M, Higham DJ, Oppo G-L (eds) The structure of financial networks. Springer, London, pp 131-163. https://doi.org/10.1007/978-1-84996-396-1_7

28. Nagurney A, Siokos S (2012) Financial networks: statics and dynamics. Advances in spatial science. Springer, Berlin

29. Caldarelli G, Battiston S, Garlaschelli D, Catanzaro M (2004) In: Ben-Naim E, Frauenfelder H, Toroczkai Z (eds) Emergence of complexity in financial networks. Springer, Berlin, pp 399-423. https://doi.org/10.1007/978-3-540-44485-5_18

30. Leitner Y (2005) Financial networks: contagion, commitment, and private sector bailouts. J Finance 60:2925-2953. https://doi.org/10.1111/j.1540-6261.2005.00821.x

31. Gai P, Kapadia S (2010) Contagion in financial networks. R Soc Lond Proc, Ser A, Math Phys Eng Sci 466:2401-2423. https://doi.org/10.1098/rspa.2009.0410

32. Battiston S, Caldarelli G (2013) Systemic risk in financial networks. J Financ Manag Mark Inst: 129-154. https://doi.org/10.12831/75568

33. Jackson MO, van den Nouweland A (2005) Strongly stable networks. Games Econ Behav 51:420-444. https://doi.org/10.1016/j.geb.2004.08.004

34. Lorenz J, Battiston S, Schweitzer F (2009) Systemic risk in a unifying framework for cascading processes on networks Eur Phys J B 71:441. https://doi.org/10.1140/epjb/e2009-00347-4

35. Amini H, Cont R, Minca A (2013) Resilience to contagion in financial networks. Math Finance 26:329-365. https://doi.org/10.1111/mafi.12051

36. Glasserman P, Young HP (2015) How likely is contagion in financial networks? J Bank Finance 50:383-399. https://doi.org/10.1016/j.jbankfin.2014.02.006

37. Bardoscia M, Battiston S, Caccioli F, Caldarelli G (2017) Pathways towards instability in financial networks. Nat Commun 8:14416. https://doi.org/10.1038/ncomms14416

38. Thurner S, Poledna S (2013) Debtrank-transparency: controlling systemic risk in financial networks. Sci Rep 3:1888. https://doi.org/10.1038/srep01888

39. Battiston S, Caldarelli G, Georg C-P, May RM, Stiglitz J (2013) Complex derivatives. Nat Phys 9:123-125. https://doi.org/10.1038/nphys2575

40. Battiston S, Caldarelli G, May RM, Roukny T, Stiglitz J (2016) The price of complexity in financial networks. Proc Nat Acad Sci USA 113:10031-10036. https://doi.org/10.1073/pnas.1521573113

41. Aït-Sahalia Y, Cacho-Diaz J, Laeven RJA (2015) Modeling financial contagion using mutually exciting jump processes. J Financ Econ 117:585-606. https://doi.org/10.1016/j.jineco.2015.03.002

42. Feinstein Z (2017) Financial contagion and asset liquidation strategies. Oper Res Lett 45:109-114. https://doi.org/10.1016/j.orl.2017.01.004

43. European Central Bank (2005) The financial risk management of the Eurosystem's monetary policy operations. Eurosystem, Frankfurt

44. Kleinberg JM (1999) Authoritative sources in a hyperlinked environment. J ACM 46:604-632 https://doi.org/10.1145/324133.324140

45. Opsahl T, Colizza V, Panzarasa P, Ramasco JJ (2008) Prominence and control: the weighted rich-club effect. Phys Rev Lett 101:168702. https://doi.org/10.1103/PhysRevLett.101.168702

\section{Submit your manuscript to a SpringerOpen ${ }^{\circ}$ journal and benefit from:}

- Convenient online submission

- Rigorous peer review

- Open access: articles freely available online

- High visibility within the field

- Retaining the copyright to your article

Submit your next manuscript at $>$ springeropen.com 